\title{
Nach dem Feuer im Bergmannsheil: Der Brandschutz entsprach den Vorschriften Interview mit Olaf Patzke
}

In den Tagen nach dem Brand im Bergmannsheil im September 2016 gab es viel Kritik an vermeintlichen Schwächen beim bauseitigen Brandschutz, selbst aus den Reihen der Feuerwehr. Ein Jahr danach entpuppt sich das Gros davon als voreilig, erklärt ein Experte der Bochumer Feuerwehr.

Zumindest laut einigen Medienberichten gab es auch seitens der Feuerwehr nach dem Brand im Bergmannsheil Ende 2016
Kritik am bauseitigen Brandschutz im Klinikum. Es hätten Sprinkleranlagen gefehlt, lautete ein Vorwurf, den Kollegen von Ihnen gemacht haben sollen.

Da gab es zum Teil Missverständnisse. Wir wissen heute klipp und klar: Die baulichen Gegebenheiten im Bergmannsheil entsprachen zum Zeitpunkt des Unglücks den Vorschriften. Sie entsprachen nicht nur den Vorschriften zum Zeitpunkt der Errichtung des Gebäudes, sondern wurden auch danach in all den Jahren fortlaufend

\section{ZUR PERSON}

Olaf Patzke (Jahrgang 1970) ist bei der Feuerwehr Bochum zuständig für den Vorbeugenden Brand- und Gefahrenschutz. Als Feuerwehrbeamter (M. Eng.) in der Abteilung Prävention ist er unmittelbar auch an der Planung des bauseitigen Brandschutzes in Kliniken beteiligt. 
an die Anforderungen angepasst. Die Brandschutztüren zum Beispiel waren funktionssicher und wirksam. Die notwendigen Brandabschnitte in mindestens der feuerbeständigen Ausführung F 90 waren vorhanden. Und Sprinkleranlagen sind eben keine generelle Vorschrift für den Bau von Kliniken.

Die Ursache des Brands war Brandstiftung, eine Patientin, die sich angezündet hat, dabei noch einen Brandbeschleuniger nutzte.

Richtig. Und gegen Brandstiftung hilft auch kein noch so umfangreiches Vorschriftenwerk.

Wir machen die regelmäßig von Bauordnungsamt und Feuerwehr begleiteten Revisionen, Checks, der Kliniken spätestens alle 3 Jahre. Und wir haben in dem Klinikum keine signifikanten Mängel festgestellt, aus denen sich konkrete Gefahrenmerkmale ergeben hätten.

Was ist mit einer Brandmeldeanlage? Die war im Bergmannsheil installiert.

Werden Sie jetzt im Nachgang des Brands den flächendeckenden Einbau von Sprinkleranlagen im Bergmannsheil fordern?

Im konkreten Fall Nein. Das ist dort nicht praktikabel. Wenn Sie in dem Gebäude heute im Nachhinein eine Sprinkleranlage einbauen wollen, führt das zu einer Sanierung, die de facto eine Entkernung des Gebäudes nötig macht. Da können Sie dann auch gleich einen Neubau hinstellen.

Nach dem Brand im Bergmannsheil gab es aber Stimmen, nach denen eine Sprinkleranlage das Feuer im Keim erstickt hätte. Solche Statements kommen allerdings oft wiederum auch von Herstellern.

In der Sache haben die Hersteller vermutlich sogar recht. Aber das ist natürlich auch eine Frage des Aufwands und der Schutzzieldefinition.

\section{Was meinen Sie?}

Wichtig ist bei einem Feuer auf jeden Fall eine zeitnahe Branddetektion durch Rauchwarnmelder, die dem Klinikpersonal eine zeitnahe Erstbrandbekämpfung erlaubt, beziehungsweise eine schnelle Räumung des betroffenen Bereiches ermög- licht. Sprinkleranlagen lösen aber in der Regel erst bei circa 68 Grad Celsius aus, und damit deutlich später als der automatische Brandmelder. Zeit ist bei einem Brandereignis in Krankenhäusern aber enorm wichtig.

Wenn Sie mich andererseits fragen, was wir bei einem Neubau ähnlicher Größe und Gebäudehöhe an gleicher Stelle heute fordern würden - Ja, da würden wir vermutlich eine Sprinkleranlage fordern.

Heißt, Neubauten sollten auch Ihrer Ansicht nach generell eine Sprinkleranlage haben?

Nein, das gilt nicht pauschal. Der Einbau von Sprinkleranlagen ist immer eine Frage des Einzelfalls. Das hängt immer von der Zugänglichkeit und der Gebäudehöhe ab.

Bei einem Bau auf der grünen Wiese, mit 2 Geschossen, mit diversen Brandabschnitten und sogenannten horizontalen Evakuierungsbereichen, komme ich unter Umständen auch ohne Sprinkleranlage aus, da ich im Notfall gut mit Löschfahrzeugen überall drankomme, und eine Brandbekämpfung insbesondere im Dachund Fassadenbereich relativ leicht und effektiv einleiten kann.

Bei einer Lage wie beim Bergmannsheil mit mehreren Geschossen und teilweise eingeschränkter Zugänglichkeit, da müssen zukünftig Alternativen gewählt werden, weil eine Brandbekämpfung im AuBenangriff durch die Feuerwehr teilweise eingeschränkt wird. Da geht es dann bei einem Neubau eher in Richtung Einbau einer Sprinkleranlage. Aber bei komplexen Bestandsbauten geht Nachrüsten nicht, das ist nicht praktikabel.

Reichen die Vorschriften zum Brandschutz in Krankenhäusern aus?

In der Praxis ja. Krankenhäuser sind als Sonderbauten brandschutztechnisch eigentlich beherrschbar, sofern die elementaren Grundprinzipien beachtet werden.

Heißt, alles ist sicher?

Absolute Sicherheit gibt es natürlich nie. Natürlich ist jeder Todesfall durch Brand einer zu viel. Aber Sie müssen auch die Relation sehen. Wir haben im Jahr in Deutschland an die 450 Todesopfer durch
Brände im privaten Wohnungsbereich zu beklagen. Im Krankenhaus sind es verglichen damit nur sehr wenige Fälle.

Also wirklich keine Erkenntnisse nach dem Brand im Bergmannsheil, dass der bauseitige Brandschutz verbessert werden muss?

Pauschal würde ich sagen Nein. Die Auflagen und Vorschriften im KrankenhausBereich sind seit Jahren hoch und haben sich bewährt. Sicherlich gibt es immer Details zu verbessern, aber die Grundstrukturen sind weitgehend konstant.

Und vergessen Sie nicht: Noch wichtiger als der oft zitierte bauseitige Brandschutz ist der betrieblich-organisatorische. Wenn das Personal auf Zack ist, wenn es den Algorithmus im Brandfall beherrscht, wenn es weiß, wie und wo die Patienten horizontal in den nächsten Brandabschnitt verschoben werden, dann ist enorm viel erreicht. Und all das hat an dem besagten Tag im Bergmannsheil gut funktioniert. Deshalb ist auch nicht mehr passiert.

Einige Experten monieren, es fehlten bundesweit einheitliche Standards für den Brandschutz in Krankenhäusern. Die Musterbauverordnung Krankenhaus ist von 1976 und damit völlig veraltet.

Die Grundlagen stehen da drin und sind auch nicht veraltet.

Sprinkler und Brandmeldeanlagen stehen nicht drinnen.

Doch, Brandmeldeanlagen und Alarmierungseinrichtungen waren da im Paragraph 25, Absatz 2 und 3 schon immer gefordert. Wir sind hier bei Krankenhäusern im Bereich Sonderbauten und da gelten in der Praxis des Baugenehmigungsprozesses bei Baugesuchen immer die schon lange etablierten Standards.

Im Übrigen kommt in NRW Ende des Jahres eine neue Bauordnung heraus. Und die Sonderbauvorschrift ist gerade im Januar 2017 neu aufgelegt worden. Das Baurecht ist also hier sicher nicht veraltet, auch nicht beim Thema Brandschutz, es wird vielmehr immer überarbeitet, gerade dann, wenn sich neue Erkenntnisse durch Brände ergeben.

Das Interview führte Bernhard Epping. 